\title{
Anion Specific Effects Drive the Formation of Li-Salt Based Aqueous Biphasic Systems
}

Nicolas Dubouis ${ }^{1,2,3}$, Arthur France-Lanord ${ }^{3,4}$, Amandine Brige ${ }^{1,5}$, Mathieu Salanne ${ }^{3,4,6^{*}}$, Alexis Grimaud ${ }^{1,2,3^{*}}$

1. Chimie du Solide et de l'Energie, Collège de France, UMR 8260, 75231 Paris Cedex 05

2. Sorbonne Université, Paris, France

3. Réseau sur le Stockage Electrochimique de l'Energie (RS2E), CNRS FR3459, 33 rue Saint Leu, 80039

Amiens Cedex, France

4. Sorbonne Université, CNRS, Physicochimie des Électrolytes et Nanosystèmes Interfaciaux, PHENIX, F75005 Paris, France;

5. Département de Chimie, Ecole normale supérieure, 75005 Paris, France

6. Institut Universitaire de France (IUF), 75231 Paris, France

\section{*Corresponding authors:}

Alexis Grimaud: alexis.grimaud@college-de-france.fr

Mathieu Salanne: mathieu.salanne@sorbonne-universite.fr 


\section{Abstract}

Aqueous biphasic systems (ABS) can form when mixing water with two compounds such as polymers, ionicliquids or simple salts. While this phenomenon has been known for decades and found applications in various fields such as biology, recycling or even more recently electrochemistry, the physics behind the formation of ABSs remains ill-understood. It was recently demonstrated that ABSs can be composed of two salts sharing the same cation ( $\mathrm{Li}^{+}$) but different anions (sulfonamide and halide). Interestingly, theirformation could not be explained by the position of the anions within the chaotropic/kosmotropic series and was rather proposed to originate from an anion size mismatch, albeit the size for these anions was never measured yet owing to the lack of a proper experimental methodology. Here, we combine experimental techniques and molecular simulations to assess the specific effects (size, shape, hydrophobic/hydrophilic character) of a series of anions and correlate them with the formation of ABSs. We demonstrate that while the anion size mismatch is a prerequisite for the formation of Li-salts based ABSs, their shape can also play an important role, providing general guidelines for forming new ABSs with potential future applications. 


\section{Introduction}

Thanks to its high dielectric constant, water is a solvent of choice to dissolve a large variety of species such as salts or polymers. When moving away from the infinite dilution regime, intriguing physico-chemical properties can occur for concentrated aqueous solutions. In the most extreme scenario where two different solutes are brought at sufficient concentration in water, the formation of an aqueous biphasic system (ABS) can occur. ${ }^{1-3}$ While this phenomenon was initially described principally for a polymer aqueous phase in contact with a salt-rich aqueous phase, recent studies revealed that such ABS can also be obtained by mixing ionic-liquids (ILS) with a salt, ${ }^{2-6}$ or simply by mixing alkali-metal salts together. ${ }^{7}$ These biphasic systems revealed promising in the field of liquid-liquid extraction, for instance. ${ }^{1,4,6}$ Their application in the field of electrochemical energy storage and conversion was also recently proposed. Hence, these ABS could prove useful to design membraneless redox-flow batteries. ${ }^{8}$ Furthermore, ABSs based on Li-salts were shown to exhibit promising properties that were used to develop sustainable and high-performance aqueous Li-ion batteries..$^{9-11}$

This recent multiplication of practical applications for ABSs urges to understand the physics driving the separation of water into two phases. Investigating the formation of ABS based on hydrophilic ILs with inorganic salts, Bridges et al. proposed that the ability of one salt to structure the water lattice around the ions (kosmotropic salt) and the other one to perturb this water network (chaotropic salt) could be at the origin for the phase separation. ${ }^{3}$ However, in a recent study, we demonstrated that mixing simple salts with a common alkali-cation, i.e. lithium chloride (LiCl) and lithium bis(trifluoromethane)sulfonimide (LiTFSI), also leads to the formation of ABSs. Both salts were shown to be kosmotrope based on viscosity measurements, which challenged the initial chaotropic-kosmotropic explanation. ${ }^{7}$ Rather, based on a model assuming ions as hard sphere charged particles, ${ }^{12}$ we proposed that a large size asymmetry between the two different anions could trigger the formation of an ABS. ${ }^{7}$ Nevertheless, this study was limited to a single halide and a single organic anion, and such assumption should be validated against a greater variety of anions. Moreover, 
the anion size mismatch was inferred from a simple chemical intuition looking at the chemical formulas of the different anions, but not directly assessed since the anion size was not measured. This therefore calls for a deeper study to check if such explanation based on the size mismatch holds true or not. In this work, we studied a series of aqueous Li-salt binary mixtures with a series of anions spanning a wide range of geometries: $\mathrm{Cl}, \mathrm{Br}, \mathrm{I}, \mathrm{NO}_{3}{ }^{-}$and $\mathrm{ClO}_{4}{ }^{-}$on the one hand, and three fluorinated sulfonimide anions on the other hand. We first determine which pairs form ABS. We then rationalize this result by using various descriptors of the anion physical properties, either determined experimentally or through DFT calculations in both the gas and liquid phases.

\section{Results \& discussion}

\section{Aqueous biphasic systems formation}

It was previously demonstrated that mixing LiCl and Li (bis(trifluoromethane)sulfonimide (TFSI) salts together with water leads to the formation of ABSs for a wide range of compositions. Thus, in order to test the influence of the geometry of the anions, we decided to first study the effect of the nature of the fluorinated sulfonimide anion on the formation of ABSs while keeping the $\mathrm{Cl}^{-}$anion for the second salt. Hence, two additional organic symmetric fluorinated anions with the following formulas $\mathrm{N}\left(\mathrm{SO}_{2}\right)_{2} \mathrm{~F}_{2}$ (bis(fluorosulfonyl)imide, $\mathrm{FSI}$ ) and $\mathrm{N}\left(\mathrm{SO}_{2} \mathrm{CF}_{2} \mathrm{CF}_{3}\right)_{2}$ (bis(pentafluoroethanesulfonyl)imide, $\mathrm{BETI}$ ) were selected from the sulfonimide family. In the first case, no formation of a biphasic solution could be spotted even for a highly concentrated solution of the two salts ( $18 \mathrm{~mol} \mathrm{~kg}^{-1}$ each). Aqueous LiFSI and LiCl thus coexist over the whole range of compositions within a single aqueous phase. In contrast, when a concentrated solution of LiBETI (15 mol kg-1) was combined with a concentrated LiCl solution (18 mol kg-1), a phase separation could be triggered. The phase diagram of the $\mathrm{LiCl}-\mathrm{LiBETI}-\mathrm{H}_{2} \mathrm{O}$ system was constructed using the cloud point method. When it is compared to the one of LiCl-LiTFSI- ${ }_{2} \mathrm{O}$ (Figure 1) a larger biphasic region is measured. 


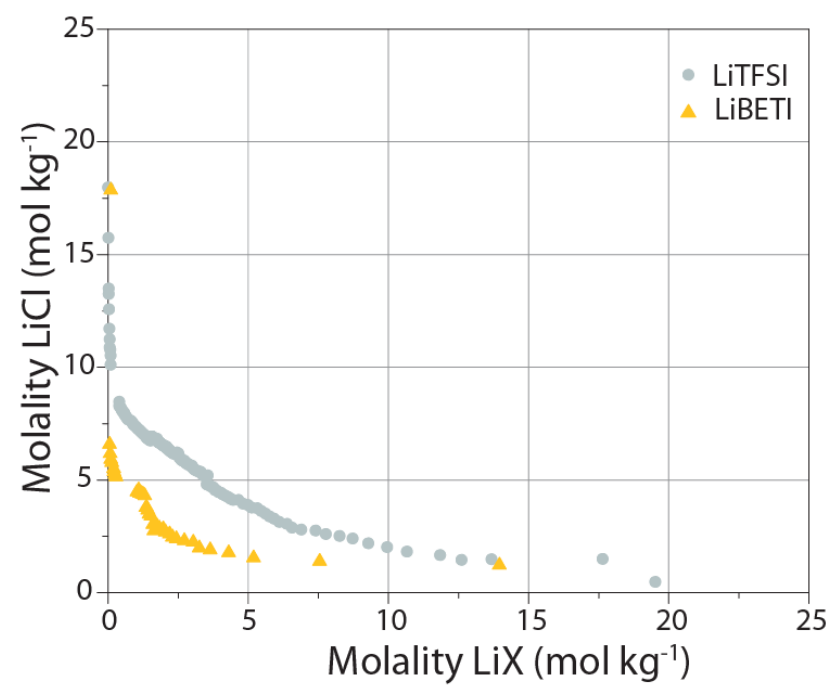

Figure 1. Phase diagrams of the $\mathrm{LiCl}-\mathrm{LiTFSI}-\mathrm{H}_{2} \mathrm{O}$ (grey circles) and $\mathrm{LiCl}-\mathrm{LiBETI}-\mathrm{H}_{2} \mathrm{O}$ (yellow triangles) systems at room temperature, constructed using the cloud point method. The regions under the lines are the monophasic regions.

In a second step, in order to test the influence of the size of the second anion on the formation of ABSs, we varied the nature of the halide $(\mathrm{X}=\mathrm{Cl}, \mathrm{Br}, \mathrm{l})$ while keeping TFSI as organic anion. In all cases, phase separations could be observed at large salt concentrations. Thus, the phase diagrams for the various LiXLiTFSI- $\mathrm{H}_{2} \mathrm{O}$ systems were constructed using the cloud point titration method. When plotted in the mass scale (Figure 2 a), the extent of the monophasic region reduces when going from $\mathrm{I}$ to $\mathrm{Br}$ and then $\mathrm{Cl}$. Nevertheless, to properly assess those physical effects at the molecular level, the molality scale ap pears more adequate. Indeed, two electrolytes sharing the same molality also share the same ion-pairs over water molecules ratios, making it possible to discuss the role of ions and solvent molecules. Interestingly, when the different phase diagrams are plotted in the molality scale (Figure $\mathbf{2}$ b), the boundary lines that separate the monophasic region from the biphasic region almost perfectly overlap for the three different halides. 
a

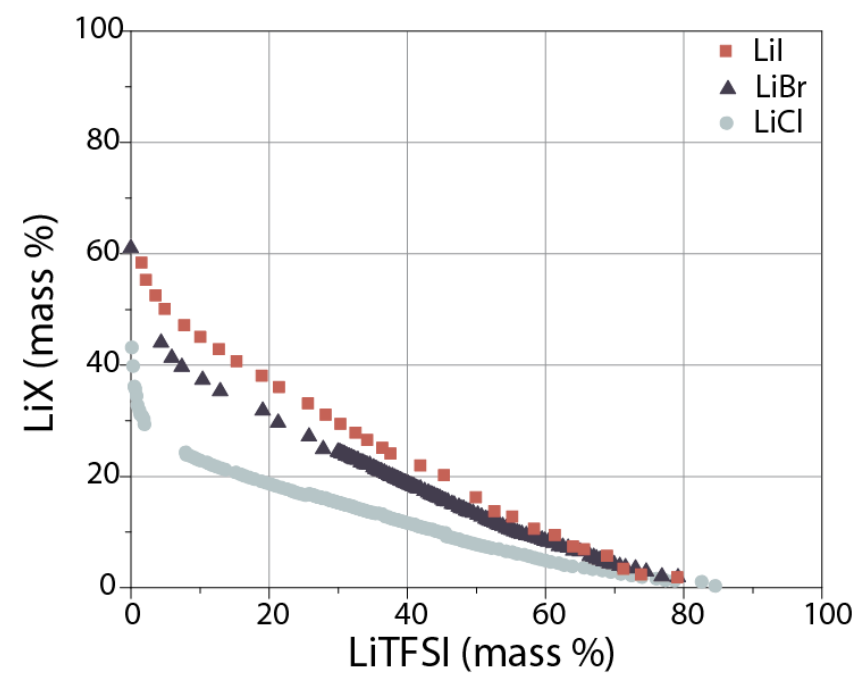

b

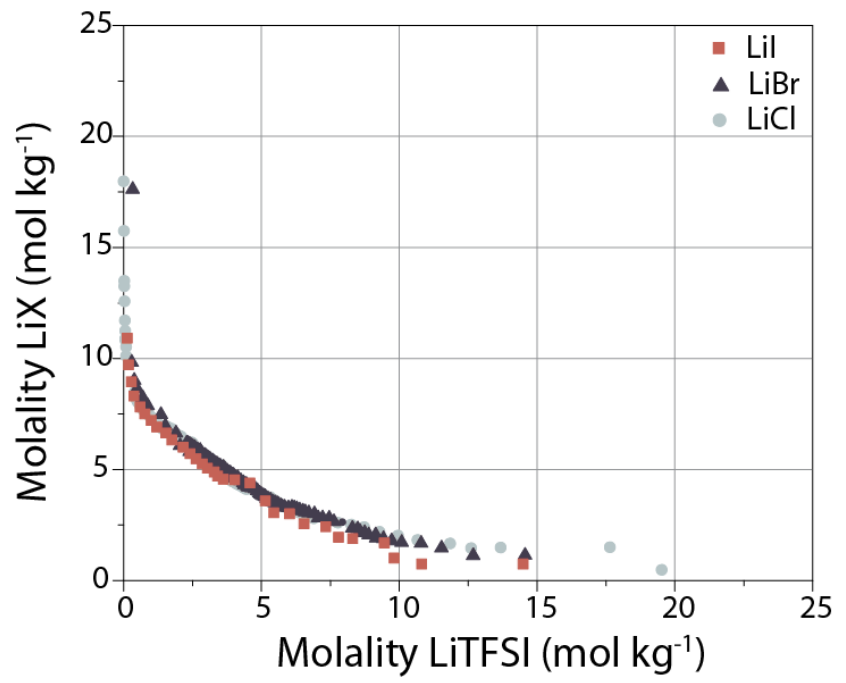

Figure 2. Phase diagram for LiX-LiTFSI- $\mathrm{H}_{2} \mathrm{O}$ systems, with $\mathrm{X}=\mathrm{Cl}$ (grey), $\mathrm{Br}$ (blue) or I (red). The phase diagrams were constructed using the cloud point method and plotted $\mathbf{a}$. in the mass scale or $\mathbf{b}$. in the molality scale. The region under the line is the monophasic region.

In order to complement the results with another family of compounds, a similar study was carried out using inorganic anions, i.e. perchlorate and nitrate, that do not belong to the inorganic halides or organic sulfonimides families and thus show different chemistries and geometries. In both cases, no ABS was formed when they were mixed with concentrated LiTFSI solutions, unlike what was previously observed for halides. The results obtained for the formation of the ABS are summarized in Table 1. In the following, we will assess the ability of various geometric descriptors to predict the occurrence of ABS. 


\begin{tabular}{|c|c|c|c|c|c|}
\cline { 2 - 6 } \multicolumn{1}{c|}{} & $\mathrm{LiCl}$ & $\mathrm{LiBr}$ & Lil & $\mathrm{LiNO}_{3}$ & $\mathrm{LiClO}_{4}$ \\
\hline LiFSI & $\mathrm{X}$ & - & - & - & - \\
\hline LiTFSI & + & + & + & $\mathrm{X}$ & $\mathrm{X}$ \\
\hline LiBETI & ++ & - & - & - & - \\
\hline
\end{tabular}

Table 1. Summary on the formation of ABS. The natures of the first and second salts are given in the columns and lines respectively. Color code: no ABS observed in black ( $\mathrm{x}$ ), formation of an ABS in yellow $(+) /$ orange $(++)$ (orange $(++)=$ smallest monophasic region), systems that have not been investigated are in grey (-).

\section{Probing the influence of the anion size from simple density measurements}

In order to be efficient, chemical descriptors should be simple to measure. Based on the theoretical model developed by Lo Celso et al., who studied the mixing behavior of charged hard spheres ${ }^{12}$ (in the absence of solvent), we expect the relative size of the two anions (or their ratio) to play an important role on the formation of an ABS. However, there is currently no consensus on the best method to determine the size of ions in solution. For example, the ion-water distances can be probed by diffraction methods or molecular simulations, ${ }_{13}^{13}$ but these two methods are rather complex to implement. A simpler approach to sense the mean ion diameters ( $\mathrm{d}_{\mathrm{ion}}$ ) of the ions composing a salt is to measure the densities of several solutions prepared with a precisely known concentration of salt and water, as described in the Materials section. ${ }^{14,15}$ The partial molar volume of an ion pair $V_{p}$ is then extrapolated by taking the intercept at $\mathrm{C}_{\mathrm{H} 2 \mathrm{O}}=0$ of a linear regression, as shown in Figure $\mathbf{3}$ a for the lithium halide salts (similar plots for sodium and potassium halides are given in the Figure S1), Figure $\mathbf{3} \mathbf{b}$ for the lithium fluorosulfonylimide salts and Figure $\mathbf{3} \mathbf{c}$ and $\mathbf{3} \mathbf{d}$ for $\mathrm{LiNO}_{3}$ and $\mathrm{LiClO}_{4}$, respectively. The mean ion diameter $\left(\mathrm{d}_{\mathrm{ion}}\right)$ is then taken as half the diameter of the sphere with a volume $V_{\text {P. }}$. The $d_{\text {ion }}$ thus obtained are given in Figure $\mathbf{3} \mathbf{e}$. 

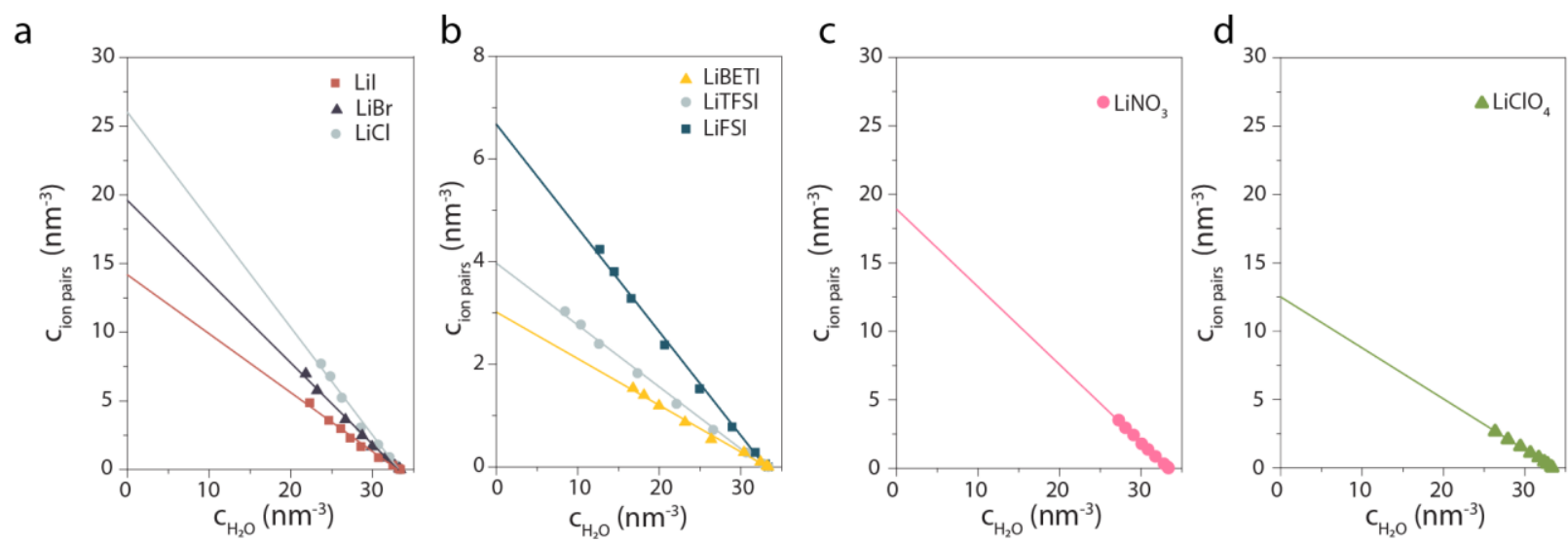

e

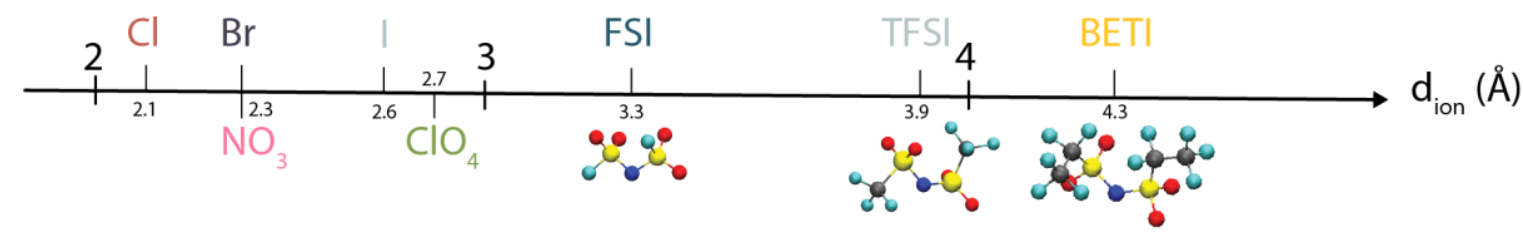

Figure 3. Determination of the anions mean ion diameter from density measurements. a. Concentration of ion pairs as a function of the water concentration for $\mathrm{LiCl}$ (grey circle), $\mathrm{LiBr}$ (blue triangle) and Lil (red squares) determined by measuring the density of solutions with different molalities. b. Concentration of ion pairs for LiFSI (blue squares), LiTFSI (grey circles) and LiBETI (yellow triangles). c. Concentration of ion pairs for $\mathrm{LiNO}_{3}$. d. Concentration of ion pairs for $\mathrm{LiClO}_{4}$. e. Mean ion diameters $(\AA)$ for all the anions computed from the apparent ion pair volume extrapolated from the concentration of ions at $\mathrm{C}_{\mathrm{H} 2 \mathrm{O}}=0$. Molecular representations are given for the organic anions with $\mathrm{N}$ atoms in dark blue, $\mathrm{S}$ atoms in yellow, $\mathrm{O}$ atoms in red, $\mathrm{C}$ atoms in black and $\mathrm{F}$ atoms in light blue.

If first looking at the large organic anion series (Figure $\mathbf{3} \mathbf{e}$ ), the size argument seems to provide a qualitatively good descriptor for the formation of the ABS. Indeed, no ABS would be formed for the LiClLiFSI- $\mathrm{H}_{2} \mathrm{O}$ system due to the small diameter of the FSI anion ( $3.9 \AA$ according to our estimate), and the larger the fluorosulfonylimide, the larger the immiscibility domain (Figure 1). However, the situation is much less clear when looking at the small inorganic anion series. Indeed, the three halides show similar phase diagrams when using the molality scale despite the increase of their radius with their atomic number (Figure $\mathbf{2} \mathbf{b}$ ). This could point towards a "threshold" size below which an ABS is formed independently of the diameter of the small ion. Nevertheless, it is worth noting that $d_{\text {ion }}(l)$ is found to be only 1.25 times larger than $d_{\text {ion }}(C l)$. This small difference, presumably not significant compared to the size of the organic TFSI anion, may explain why no effect of the halide size is observed on the phase diagrams. 
However, as seen on Figure $\mathbf{3} \mathbf{e}$, the diameters of the nitrate and perchlorate anions are very similar to the ones of $\mathrm{Br}$ and $\mathrm{r}$, respectively, which is either due to a poor determination of the ion diameter or to the failure of ion diameter as a sole descriptor for the formation of ABS. Concerning the first hypothesis, it is certain that the diameters obtained herein from density measurements are approximate. For example, they are twice smaller than those of the same anions within pure ionic crystals. ${ }^{16}$ However, this observation surely results from the small size of the Li cation ( $d=1.80 \AA$ in pure ionic crystals $)^{16}$, even compared to those of the halides (at least twice larger), which results in a systematic underestimation of $d_{i o n}$ for the anions. Indeed, the $d_{\text {ion }}$ value estimated by this method is the average ion diameter for a given ion-pair, e.g. considering similarly the anion and the cation. In order to assess this effect, the diameter was also estimated using density measurements of sodium halide and potassium halide salts dissolved in water (Figure S1). The diameters are almost invariant between LiX, $\mathrm{NaX}$ and $\mathrm{KX}$ salts (X being the halides) while the cation size should increase, thus showing that the anion contribution to the $d_{\text {ion }}$ largely dominates the one of the cation and validating this experimental method as a good estimation of the relative anion sizes. In addition, a spherical geometry for the ion-pairs was assumed in order to determine the $d_{i o n}$. While this assumption may roughly hold for simple spherical anions such as the halides, it probably fails for ions with more complex geometries. Indeed, the $\mathrm{NO}_{3}{ }^{-}$anion has a triangular shape while the $\mathrm{ClO}_{4}$ one forms a tetrahedron. It is thus possible that the $\mathrm{Li}^{+}$ ions can approach closer to the anion center along specific directions (for example between the two tips of the triangle in the case of the nitrate), thus resulting in an underestimation of the anion diameter using the density measurement method. It is probably even more irrelevant for the organic anions since their spatial conformation may depend on their concentration. Indeed, it was demonstrated that in so-called water-insalt electrolyte (LiTFSI at $20 \mathrm{~mol} \mathrm{~kg}^{-1}$ ) developed for aqueous Li-ion batteries, the formation of contact ionpairs and ion-aggregates at such large ionic concentration leads to different conformation of the TFSI anions. ${ }^{17}$ Such observation further highlights the difficulties in determining which characteristic distance should be used to describe ionic sizes (interatomic distances, radius, etc.). This calls for the use of more involved descriptors to analyze the formation of ABSs when the anions' characteristic sizes are too similar. 


\section{Probing the influence of the anion hydrophobicity using gas phase DFT calculations}

Further complicating such study, an analysis solely based on the ion size also misses features such as differences in hydrophobicity between anions. Originally, ABS formation was thought to be determined by the structure-forming (kosmotropic) or structure-breaking (chaotropic) nature of the ions; however, we recently showed that such a criterion is not sufficient. ${ }^{7}$ An ion's structure making or breaking behavior is intimately related to how an ion interacts with its surroundings. In the case of a dilute aqueous solution, this is generally characterized by the hydrophilic or hydrophobic character of an ion. We therefore investigated if differences in the anions' hydrophobicity behavior could rationalize the cases for which the sole difference in anions size cannot explain the ABS formation.

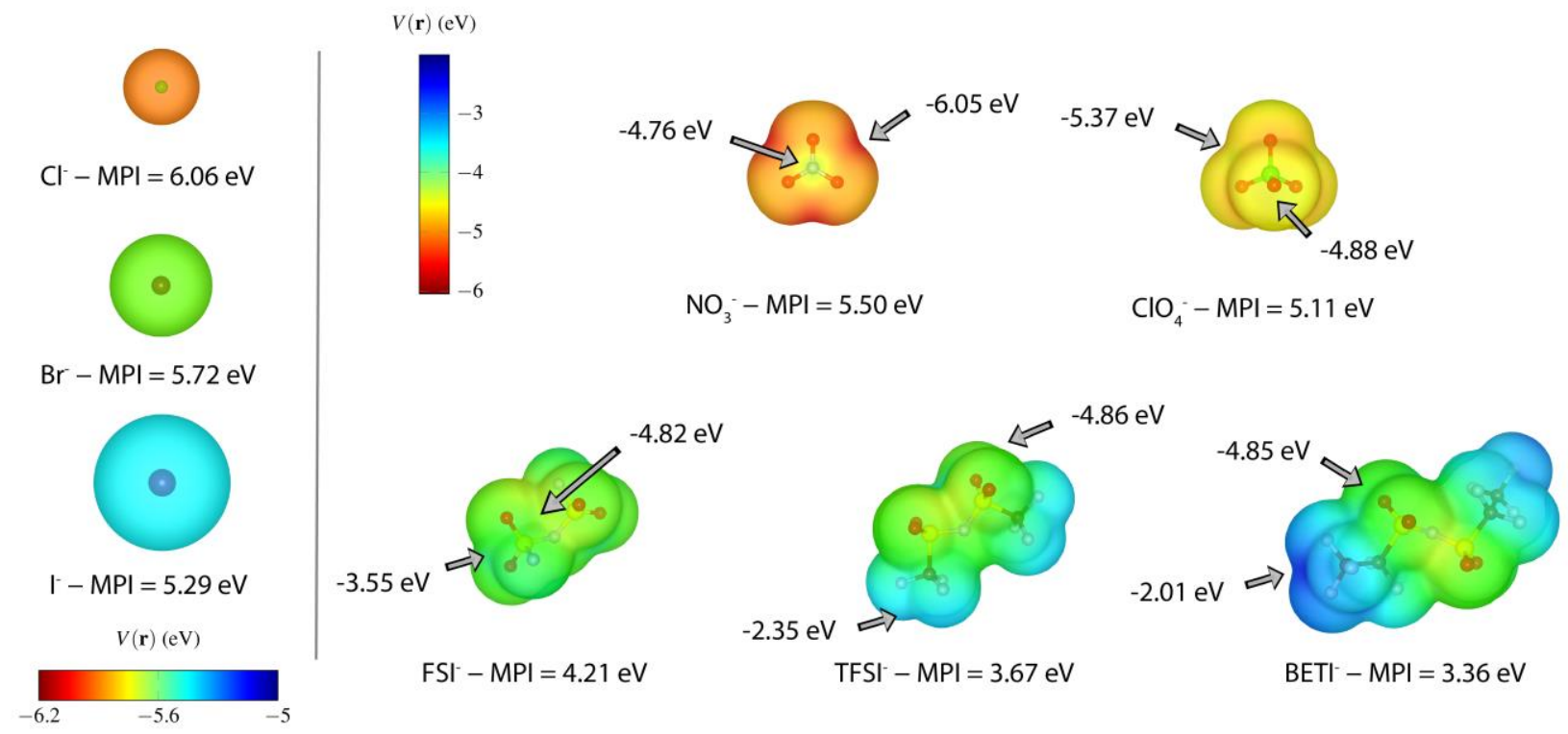

Figure 4. Electrostatic potential $V(\boldsymbol{r})$ mapped to the $0.001 \mathrm{e} / \AA^{3}$ isosurface of the electron density for a series of anions $\left(\mathrm{Cl}^{-}, \mathrm{Br}, \mathrm{l}\right.$; $\left.\mathrm{NO}_{3}{ }^{-}, \mathrm{ClO}_{4}^{-}, \mathrm{FSI}, \mathrm{TFSI}, \mathrm{BETI}\right)$ obtained from gas phase quantum chemistry calculations, along with the molecular polarity index (MPI). For non-spherical anions, extrema values of the electrostatic potentials are indicated along with their location on the isosurfaces.

To do so, the simplest approach would consist in using the hydration free energies of the anions as a descriptor. However, experimental measurements show significant variability which can be attributed to the various assumptions required for extrapolating such values ${ }^{18}$ Nevertheless, the experimentally determined Gibbs free energy of hydration ${ }^{19}$ do place $\mathrm{NO}_{3}$ as more hydrophilic than $\mathrm{I}$, which seems to indicate that this 
descriptor is also deficient for explaining their different abilities to form ABSs. Molecular polarity index (MPI) obtained from the electrostatic potential calculated at the quantum chemistry level has also been proposed to correlate with the ion hydrophilicity, the larger value of the MPI corresponding to a more hydrophilic character. ${ }^{20}$ Results obtained for MPI computed for the different anions are shown on Figure 4. Here again, it is clear that this descriptor is not able to catch differences between the $\mathrm{Br}, \mathrm{NO}_{3}^{-}, \mathrm{I}^{-}$and $\mathrm{ClO}_{4}^{-}$since they all display very similar MPIs.

\section{Probing the solvated anions geometry using ab initio molecular dynamics simulations}

In order to clarify the differences existing between halides and perchlorate or nitrate ions, we then computationally investigated the shape of these ions in solution. For that, ab initio molecular dynamics simulations were performed in order to avoid any pitfall associated to the choice of a particular force field. The computed radial distribution functions between the halide anion or the central atom of the nitrate and perchlorate anion and the hydrogen atoms from the water molecules are shown Figure 5. We observe that this function hints towards a larger diameter for the $\mathrm{NO}_{3}^{-}$anion compared to our experimental estimate, since it is almost superimposed with the one of the I anion (while density measurements gave similar diameters for $\mathrm{NO}_{3}{ }^{-}$and $\mathrm{Br}$, as shown Figure 3). In addition, the $\mathrm{ClO}_{4}^{-}$anion displays the largest preferred distance to the water molecules. However, the differences observed here are too small to conclude further on a diameter effect. 


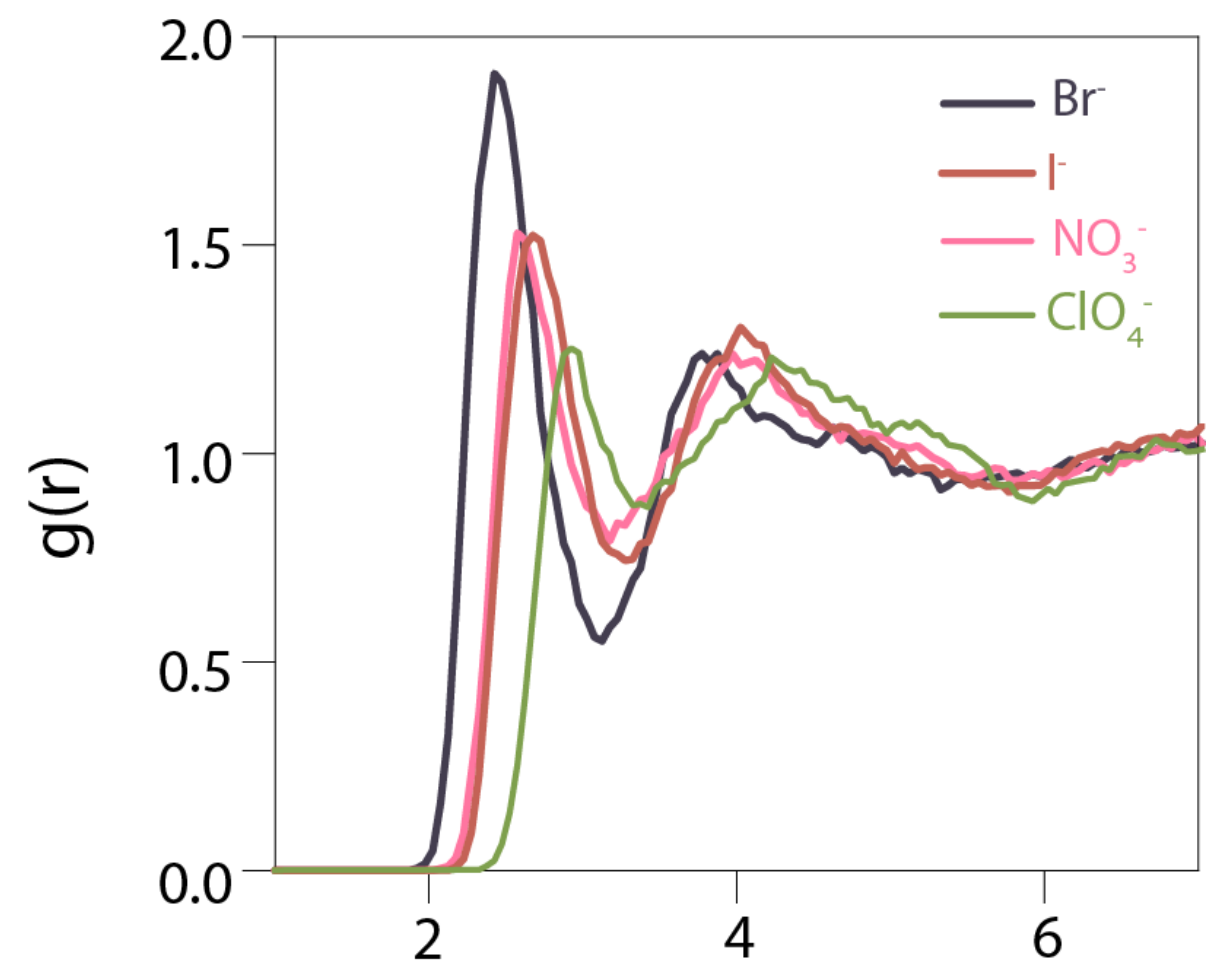

Figure 5. Radial distribution function between the halide anion or the central atom of the nitrate and perchlorate anion and the hydrogen atoms from the water molecules.

Molecular dynamics simulations allow us to go further and determine the shape of the solvation shell. This was done using a domain decomposition analysis of the trajectory, using the Voronoi tessellation method. ${ }^{21}$ Doing so, the shape of the anions could be characterized, assuming the anion to occupy the ensemble of Voronoi cells containing the anion and no other atom from the local environment. Results are presented in Table 2. It is worth noting that the surface area and volume of the calculated domains are only estimated since they strongly depend on the metrics used as Voronoi radii (in our case, van der Waals radii). However, the use of different parameters would lead to qualitatively similar results. It is therefore clear that, owing to their molecular character, the nitrate and perchlorate anions occupy significantly more space than their halides counterparts. This result therefore reinforces the argument following which ABS are formed when the two anions have different sizes due to packing constraints, albeit this cannot always be cast in a simple descriptor as initially attempted in this work. 


\begin{tabular}{|c|c|c|c|c|}
\hline Anion & $\mathbf{S}\left(\AA^{2}\right)$ & $\mathbf{V}\left(\AA^{3}\right)$ & $\mathbf{Q}$ & $\mathbf{N}$ \\
\hline $\mathrm{Br}$ & $62.68 \pm 2.93$ & $38.70 \pm 2.41$ & $0.829 \pm 0.020$ & $28.4+4.0$ \\
\hline $\mathrm{NO}_{3}^{-}$ & $93.26 \pm 4.44$ & $61.87 \pm 4.00$ & $0.731 \pm 0.020$ & $70.4 \pm 5.7$ \\
\hline $\mathrm{r}$ & $70.08 \pm 2.57$ & $47.24 \pm 2.24$ & $0.856 \pm 0.016$ & $32.4 \pm 3.7$ \\
\hline $\mathrm{ClO}_{4}^{-}$ & $114.46 \pm 4.57$ & $84.14 \pm 4.33$ & $0.731 \pm 0.023$ & $85.8 \pm 5.6$ \\
\hline
\end{tabular}

Table 2. Geometrical characteristics of the anion-containing domains: surface area $(S)$, volume $(V$, isoperimetric quotient $(Q)$, and number of facets $(M$. Reported values are averages with associated standard deviations calculated over the trajectories.

A third important quantity which can be extracted is the sphericity of the calculated domain, through the introduction of the isoperimetric quotient $Q$ defined as:

$$
Q=36 \pi \frac{V^{2}}{A^{3}}
$$

For a spherical domain, $Q=1$, while the asphericity increases with decreasing $Q$ value. As seen in Table 2, halide domains show high $Q$ values. Such a result is not trivial, as this method probes the imprint of the anion in its environment rather than the shape of its electronic cloud. In details, the l' domain is slightly more spherical than the $\mathrm{Br}$ one, mostly because it is larger and composed of more facets ( $\mathrm{N}$ values in Table 2). On the other hand, both nitrate and perchlorate domains show significantly larger asphericities. Since TFSI anion exhibits a highly non-spherical geometry, its Q value should certainly be low as well, explaining its mixing with low Q-values anions such as $\mathrm{ClO}_{4}^{-}$or $\mathrm{NO}_{3}^{-}$and the formation of $\mathrm{ABS}$ in presence of highly spherical halide anions. Overall, this means that this quantity may be seen as a secondary descriptor for systems in which the diameter is not sufficient. 


\section{Conclusion}

From this study, we can conclude that a large size asymmetry between different anions is a prerequisite to form ABSs from Li-salts. The size of the largest (organic) anion appears to be critical to determine how "easy" it is for two salts to segregate into two coexisting aqueous phases, but the results are less clear concerning the impact of the size of the smallest (inorganic) anion. The comparison between $\mathrm{Br}^{-} \mathrm{I}^{-} \mathrm{NO}_{3}^{-}$and $\mathrm{ClO}_{4}^{-}$, in which the two halides form ABSs with LiTFSI while the two others don't, shows that the local interaction with the water molecules, as described by their hydrophilic character or through the corresponding radial distribution functions, does not play a crucial role. Instead, a geometrical analysis of the solvation shell shows important differences between the two pairs of ions in terms of occupied volume and asphericity, which hints towards an important role of the packing ability of the ions, hence their overall shape, on the formation of ABSs.

\section{Material and methods}

\section{Materials}

The following salts LiCl (anhydrous 98+, Alfa Aesar), LiBr (anhydrous 99+, Alfa Aesar), Lil (anhydrous, 99+Alfa Aesar), LiTFSI (Solvay), LiBETl (TCl Chemicals), NaCl (99.0\% min, Sigma-Aldrich), NaBr (99+, water < 1\%, Alfa Aesar), Nal (99.5\% min, Alfa), KCl (99\%, Alfa Aesar), KBr (FT-IR grade, > 99\%, Sigma-Aldrich) and KI (Alfa Aesar) were used without further purification. However, solutions prepared with LiFSI (TCl Chemicals) were showing some turbidity and were then centrifuged before being used in order to remove the insoluble impurities. Ultrapure water (Mili-Q) was used to prepare all the solutions. 


\section{Phase Diagram Construction}

The phase diagrams were constructed using the cloud point titration method as previously described? Briefly, to a solution with a known amount of a salt and water, a perfectly known mass of the other salt aqueous solution is added until a cloudy solution is obtained after being vortexed. Then, water is added dropwise till the solution becomes clear again. After weighing the water added, the first step is reproduced. Generally, the diagrams were constructed in two times: first, the solution containing the inorganic salt is added to the one containing the organic salt and after, the reverse operation is conducted.

\section{Cion Vs. $\mathrm{CH}_{2} \mathrm{O}$ plot construction}

The densities $d$ of solution containing a precisely know amount of a salt $\left(m_{\text {salt }}\right)$ and water $\left(m_{\mathrm{H}_{2} \mathrm{O}}\right)$ were measured with an electronic densitometer (Anton Paar, DMA 35 Basic). Thus the $c_{\text {salt }}$ and $c_{\mathrm{H}_{2} \mathrm{O}}$ were extracted following the equations: $c_{\text {salt }}=\frac{d}{M_{\text {salt }}\left[\frac{m_{H_{2} O}}{m_{\text {salt }}+1}\right]}$ and $c_{H_{2} O}=\frac{d}{M_{H_{2} O} O\left[\frac{m_{\text {salt }}}{m_{H_{2} O} \mathrm{O}}\right]}$ and were further converted to the $\mathrm{nm}^{-3}$ scale.

\section{Condensed phase ab initio molecular dynamics calculations}

Initial configurations composed of one anion and 111 water molecules were generated using a Monte Carlo procedure implemented in the packmol ${ }^{22}$ program, with densities set to the experimental value at the corresponding $\mathrm{LiX}\left(\mathrm{X}=\mathrm{Br}, \mathrm{I}, \mathrm{NO}_{3}, \mathrm{ClO}_{4}{ }^{-}\right)$concentrations. All subsequent calculations were performed using Density Functional Theory (DFT) within the Generalized Gradient Approximation (GGA) in the form of the rev-PBE exchange-correlation functional. ${ }^{23} \mathrm{~A}$ correction to dispersion interactions in the form of the D3 method $^{24}$ was applied. We adopted a representation in the form of a dual basis of atom-centered Gaussian orbitals and plane waves (GPW), as implemented in the QUICKSTEP package of the CP2K program. ${ }^{25} \mathrm{~A}$ grid cutoff of 500 Ry with an associated relative cutoff of 50 Ry were found enough to converge the total energy, using molecule-optimized short-range double-zeta valence plus polarization basis sets, and GTH pseudopotentials (DZVP-MOLOPT-SR-GTH). The Kohn-Sham equations were solved self-consistently using 
the Orbital Transformation (OT) method, with a convergence criterion set to $10^{-5}$. For each system, we performed a 72 ps - long Born-Oppenheimer molecular dynamics simulation. We used the deuterium mass for hydrogen, in order to guarantee a satisfying energy conservation using a time step of $0.5 \mathrm{fs}$. Systems were propagated in the canonical ensemble, using a Nosé-Hoover chain of three thermostats with a time constant set to $50 \mathrm{fs}$. The first $12 \mathrm{ps}$ were discarded as equilibration, according to the time evolution of the total energy of the systems; the subsequent 60 ps were used to calculate radial distribution functions associated to the anion hydration. Voronoi tessellation was performed using the TRAVIS program ${ }^{262}$ on the sampled dynamics.

\section{Gas phase quantum chemistry calculations}

All gas phase calculations were performed using the all-electron ORCA program. ${ }^{27,28}$ We have used the PBE0 hybrid functional ${ }^{29}$ with D3 correction, along with minimally-augmented triple-zeta valence plus polarization basis sets (ma-def2-TZVP). All anion geometries were optimized, with tight convergence criteria, which also ap plies to the SCF convergence ("TightSCF" keyword). The converged wavefunctions were further post-processed using the Multiwfn program ${ }^{30}$ to compute the molecular polarity indexs (MPI), defined as:

$$
M P I=\frac{1}{A} \iint_{S}|V(\boldsymbol{r})| d S,
$$

where $A$ is the area of the 0.001 e/ $\AA^{3}$ isosurface of the electron density $(S)$, and $V(\boldsymbol{r})$ is the electrostatic potential. The electrostatic potential maps were rendered using the VESTA program. ${ }^{31}$

\section{Acknowledgments}

N.D. acknowledges the Ecole normale supérieure for his PhD. scholarship. We acknowledge the French National Research Agency for its support through the Labex STORE-EX (ANR-10LABX-76-01) and Project 
BALWISE (ANR-19-CE05-0014). This work was granted access to the HPC resources of CINES under Allocation A0080910463 made by $\mathrm{GENCl}$. This project received funding from the European Research Council under the European Union's Horizon 2020 Research and Innovation Programme (Grant 771294).

\section{Competing interests}

The authors declare no competing interests

\section{References}

(1) Freire, M. G.; Cláudio, A. F. M.; Araújo, J. M. M.; Coutinho, J. A. P.; Marrucho, I. M.; Lopes, J . N. C.; Rebelo, L. P. N. Aqueous Biphasic Systems: A Boost Brought about by Using lonic Liquids. Chem. Soc. Rev. 2012, 41 (14), 4966-4995. https://doi.org/10.1039/C2CS35151].

(2) Gutowski, K. E.; Broker, G. A.; Willauer, H. D.; Huddleston, J. G.; Swatloski, R. P.; Holbrey, J. D.; Rogers, R. D. Controlling the Aqueous Miscibility of lonic Liquids: Aqueous Biphasic Systems of Water-Miscible Ionic Liquids and Water-Structuring Salts for Recycle, Metathesis, and Separations. Journal of the American Chemical Society 2003, 125(22), 6632-6633. https:// doi.org/10.1021/ja0351802.

(3) Bridges, N. J .; Gutowski, K. E.; Rogers, R. D. Investigation of Aqueous Biphasic Systems Formed from Solutions of Chaotropic Salts with Kosmotropic Salts (Salt-Salt ABS). Green Chem. 2007, 9 (2), 177183. https://doi.org/10.1039/B611628K.

(4) Pei, Y.; Wang, J.; Liu, L.; Wu, K.; Zhao, Y. Liquid-Liquid Equilibria of Aqueous Biphasic Systems Containing Selected Imidazolium lonic Liquids and Salts. J. Chem. Eng. Data 2007, 52 (5), 20262031. https://doi.org/10.1021/je700315u.

(5) Freire, M. G.; Louros, C. L. S.; Rebelo, L. P. N.; Coutinho, J. A. P. Aqueous Biphasic Systems Composed of a Water-Stable lonic Liquid + Carbohydrates and Their Applications. Green Chem. 2011, 13(6), 1536-1545. https://doi.org/10.1039/C1GC15110] .

(6) Ventura, S. P. M.; Neves, C. M. S. S.; Freire, M. G.; Marrucho, I. M.; Oliveira, J.; Coutinho, J. A. P. Evaluation of Anion Influence on the Formation and Extraction Capacity of lonic-Liquid-Based Aqueous Biphasic Systems. J. Phys. Chem. B2009, 113(27), 9304-9310. https://doi.org/10.1021/jp903286d.

(7) Dubouis, N.; Park, C.; Deschamps, M.; Abdelghani-Idrissi, S.; Kanduč, M.; Colin, A.; Salanne, M.; Dzubiella, J:; Grimaud, A.; Rotenberg, B. Chasing Aqueous Biphasic Systems from Simple Salts by Exploring the LiTFSI/LiCl/H2O Phase Diagram. ACS Cent. Sci. 2019, 5(4), 640-643. https://doi.org/10.1021/acscentsci.8b00955.

(8) Navalpotro, P.; Neves, C. M. S. S.; Palma, J.; Freire, M. G.; Coutinho, J . A. P.; Marcilla, R. Pioneering Use of Ionic Liquid-Based Aqueous Biphasic Systems as Membrane-Free Batteries. Advanced Science 2018, 5(10), 1800576. https://doi.org/10.1002/advs.201800576. 
(9) Yang, C.; Suo, L.; Borodin, O.; Wang, F.; Sun, W.; Gao, T.; Fan, X.; Hou, S.; Ma, Z.; Amine, K.; Xu, K.; Wang, C. Unique Aqueous Li-Ion/Sulfur Chemistry with High Energy Density and Reversibility. Proc Natl Acad Sci USA 2017, 114(24), 6197-6202. https://doi.org/10.1073/pnas.1703937114.

(10) Yang, C.; Chen, J.; J, X.; Pollard, T. P.; Lü, X.; Sun, C.-J.; Hou, S.; Liu, Q.; Liu, C.; Qing, T.; Wang, Y.; Borodin, O.; Ren, Y.; Xu, K.; Wang, C. Aqueous Li-Ion Battery Enabled by Halogen Conversion-Intercalation Chemistry in Graphite. Nature 2019, 569 (7755), 245-250. https://doi.org/10.1038/s41586-019-11756.

(11) Serva, A.; Dubouis, N.; Grimaud, A.; Salanne, M. Confining Water in lonic and Organic Solvents to Tune Its Adsorption and Reactivity at Electrified Interfaces. Acc. Chem. Res. 2021, 54 (4), 1034-1042. https://doi.org/10.1021/acs.accounts.0c00795.

(12) Lo Celso, F.; Triolo, A.; Triolo, R. Phase Separation in Multi-Component Mixtures: The FourComponent Case. Physica A: Statistical Mechanics and its Applications2002, 304(1), 299-307. https://doi.org/10.1016/S0378-4371(01)00511-8.

(13) Marcus, Y. Ionic Radii in Aqueous Solutions. 24.

(14) Smith, A. M.; Lee, A. A.; Perkin, S. The Electrostatic Screening Length in Concentrated Electrolytes Increases with Concentration. J. Phys. Chem. Lett. 2016, 7(12), 2157-2163. https://doi.org/10.1021/acs.jpclett.6b00867.

(15) Coles, S. W.; Park, C.; Nikam, R.; Kanduč, M.; Dzubiella, J.; Rotenberg, B. Correlation Length in Concentrated Electrolytes: Insights from All-Atom Molecular Dynamics Simulations. J. Phys. Chem. $B$ 2020, 124 (9), 1778-1786. https:// doi.org/10.1021/acs.jpcb.9b10542.

(16) Shannon, R. D. Revised Effective lonic Radii and Systematic Studies of Interatomic Distances in Halides and Chalcogenides. Acta Cryst A 1976, 32(5), 751-767. https://doi.org/10.1107/S0567739476001551.

(17) Suo, L.; Borodin, O.; Gao, T.; Olguin, M.; Ho, J.; Fan, X.; Luo, C.; Wang, C.; Xu, K. "Water-in-Salt" Electrolyte Enables High-Voltage Aqueous Lithium-Ion Chemistries. Science 2015, 350 (6263), 938 943. https://doi.org/10.1126/science.aab1595.

(18) Lamoureux, G.; Roux, B. Absolute Hydration Free Energy Scale for Alkali and Halide lons Established from Simulations with a Polarizable Force Field. J. Phys. Chem. B2006, 110 (7), 3308-3322. https://doi.org/10.1021/jp056043p.

(19) Ambrosone, L.; Andini, S.; Castronuovo, G.; Elia, V.; Guarino, G. Empirical Correlations between Thermodynamic and Spectroscopic Properties of Aqueous Solutions of Alkan-m,n-Diols. Excess Enthalpies and Spin-Lattice Relaxation Times at 298.15 K. J. Chem. Soc., Faraday Trans. 1991, 87(18), 2989-2993. https://doi.org/10.1039/FT9918702989.

(20) Sun, W.; Wang, F.; Zhang, B.; Zhang, M.; Küpers, V.; Ji, X.; Theile, C.; Bieker, P.; Xu, K.; Wang, C.; Winter, M. A Rechargeable Zinc-Air Battery Based on Zinc Peroxide Chemistry. Science 2021, 371 (6524), 46-51. https://doi.org/10.1126/science.abb9554.

(21) Brehm, M.; Weber, H.; Thomas, M.; Hollóczki, O.; Kirchner, B. Domain Analysis in Nanostructured Liquids: A Post-Molecular Dynamics Study at the Example of lonic Liquids. ChemPhysChem 2015, 16(15), 3271-3277. https://doi.org/10.1002/cphc.201500471.

(22) Martínez, L.; Andrade, R.; Birgin, E. G.; Martínez, J. M. PACKMOL: A package for building initial configurations for molecular dynamics simulations. J. Comput. Chem. 2009, 30 (13), 2157-2164. https://doi.org/10.1002/jcc.21224.

(23) Zhang, Y.; Yang, W. Comment on "Generalized Gradient Approximation Made Simple". Phys. Rev. Lett. 1998, 80 (4), 890-890. https://doi.org/10.1103/PhysRevLett.80.890.

(24) Grimme, S.; Antony, J.; Ehrlich, S.; Krieg, H. A Consistent and Accurate Ab Initio Parametrization of Density Functional Dispersion Correction (DFT-D) for the 94 Elements H-Pu. J. Chem. Phys. 2010, 132 (15), 154104. https://doi.org/10.1063/1.3382344.

(25) Kühne, T. D.; lannuzzi, M.; Del Ben, M.; Rybkin, V. V.; Seewald, P.; Stein, F.; Laino, T.; Khaliullin, R. Z.; Schütt, O.; Schiffmann, F.; Golze, D.; Wilhelm, J.; Chulkov, S.; Bani-Hashemian, M. H.; Weber, V.; 
Borštnik, U.; Taillefumier, M.; Jakobovits, A. S.; Lazzaro, A.; Pabst, H.; Müller, T.; Schade, R.; Guidon, M.; Andermatt, S.; Holmberg, N.; Schenter, G. K.; Hehn, A.; Bussy, A.; Belleflamme, F.; Tabacchi, G.; Glöß, A.; Lass, M.; Bethune, I.; Mundy, C. J .; Plessl, C.; Watkins, M.; VandeVondele, J.; Krack, M.; Hutter, J. CP2K: An Electronic Structure and Molecular Dynamics Software Package - Quickstep: Efficient and Accurate Electronic Structure Calculations. J. Chem. Phys. 2020, 152 (19), 194103. https://doi.org/10.1063/5.0007045.

(26) Brehm, M.; Kirchner, B. TRAVIS - A Free Analyzer and Visualizer for Monte Carlo and Molecular Dynamics Trajectories. J. Chem. Inf. Model. 2011, 51 (8), 2007-2023. https://doi.org/10.1021/ci200217w.

(27) Neese, F. The ORCA Program System. WIREs Computational Molecular Science 2012, 2(1), 73-78. https://doi.org/10.1002/wcms.81.

(28) Neese, F. Software Update: The ORCA Program System, Version 4.0. WIREs Computational Molecular Science 2018, 8(1), e1327. https://doi.org/10.1002/wcms.1327.

(29) Adamo, C.; Barone, V. Toward Reliable Density Functional Methods without Adjustable Parameters: The PBEO Model. J. Chem. Phys. 1999, 110 (13), 6158-6170. https://doi.org/10.1063/1.478522.

(30) Lu, T.; Chen, F. Multiwfn: A Multifunctional Wavefunction Analyzer. Journal of Computational Chemistry 2012, 33(5), 580-592. https://doi.org/10.1002/jcc.22885.

(31) Momma, K.; Izumi, F. VESTA 3 for Three-Dimensional Visualization of Crystal, Volumetric and Morphology Data. J App/Cryst 2011, 44 (6), 1272-1276. https://doi.org/10.1107/S0021889811038970. 